\title{
Analysis of Femtosecond Timing Noise and Stability in Microwave Components
}

\author{
Michael R. Whalen \\ Office of Science, Science Undergraduate Laboratory Internship Program
}

Stevens Institute of Technology

SLAC National Accelerator Laboratory

Stanford, CA

August 14, 2010

Prepared in partial fulfillment of the requirement on the Office of Science, Department of Energy's Science Undergraduate Laboratory Internship under the direction of JosefFrisch at the LCLS Diagnostics Group at SLAC National Accelerator Laboratory.

Participant:

Signature

Research Advisor:

Signature 


\section{Table of Contents}

Abstract

Introduction

Materials and Methods

2

Results

7

Disc ussion-Sensitivity

Acknowledgments

References

11

Figures and Tables 


\begin{abstract}
Analysis of Femtosecond Timing Noise and Stability in Microwave Components. MICHAEL WHALEN (Stevens Institute of Technolo gy, Hoboken, NJ 07030) JOSEPH FRISCH (LCLS Diagnostics Group, SLAC National Accelerator Laboratory, Stanford CA, 94025)
\end{abstract}

To probe chemical dynamics, X-ray pump-probe experiments trigger a change in a sample with an optical laser pulse, followed by an X-ray probe. At the Linac Coherent Light Source, LCLS, timing differences between the optical pulse and $\mathrm{x}$-ray probe have been observed with an accuracy as low as 50 femtoseconds. This sets a lower bound on the number of frames one can arrange over a time scale to recreate a "movie" of the chemical reaction. The timing system is based on phase measurements from signals corresponding to the two laser pulses; these measurements are done by using a double-balanced mixer for detection. To increase the accuracy of the system, this paper studies parameters affecting phase detection systems based on mixers, such as signal input power, noise levels, temperature drift, and the effect these parameters have on components such as the mixers, splitters, amplifiers, and phase shifters. Noise data taken with a spectrum analyzer show that splitters based on ferrite cores perform with less noise than stripline splitters. The data also shows that noise in specific mixers does not correspond with the changes in sensitivity per input power level. Temperature drift is seen to exist on a scale between 1 and $27 \mathrm{fs} /{ }^{\circ} \mathrm{C}$ for all of the components tested. Results show that any components using more metallic conductor tend to exhibit more noise as well as more temperature drift. The scale of these effects is large enough that specific care should be given when choosing components and designing the housing of high precision microwave mixing systems for use in detection systems such as the LCLS. With these improvements, the timing accuracy can be improved to lower than currently possible. 


\section{Introduction}

The Linac Coherent Light Source (LCLS) currently produces powerful X-ray pulses with energies up to $10 \mathrm{KeV}$. The ability to produce such bright light beams at these wavelengths has given the scientific community a remarkable tool for probing molecules down to the resolution of single atoms. At the LCLS, X-ray pump-probe experiments are carried out to probe the dynamics of molecules. A short, intense optical laser pulse will initiate the molecular change or reaction, followed by an LCLS X-ray pulse used for scattering and analysis. By varying and noting the timing difference between the laser pulse and the x-ray pulse, one can repeat the experiment numerous times with different separations and reconstruct a "movie" of the reaction.

Currently, the LCLS can produce x-ray pulses as short as five femtoseconds. This puts an absolute lower bound on the phase timing difference that can be measured. To determine the timing difference between the optical laser and x-ray pulses, a measurement system consisting of feedback mechanisms and phase shifters is in place. This is to account for cable length differences and small phase drifts between the components. All timing is derived from the same master oscillator, which is used to first accelerate the electrons. Due to the structure of the accelerator, these electrons travel along locked in phase with one crest of the source. Once they reach relativistic speeds, they pass through the undulators, creating a coherent x-ray source. The master source also drives the optical laser pulse, which is shifted to arrive at the same time as the X-ray pulse. To do this, the traveling electron bunch passes through an S-band radio frequency (RF) cavity, which resonates and creates an electric signal. This signal enters the phase detector, which is a mixer, along with the master oscillator signal, and is adjusted by the phase shifter to zero the mixer output, which is the region where the readings are most sensitive. With these current microwave techniques, physicists at Stanford Linear Accelerator Center (SLAC) have

been able to measure timing separations with an accuracy of 50 femtoseconds ${ }^{[1]}$. The two main 
concerns with this measurement system are noise levels and drift stability of the electronic devices. Along with the mixers, other microwave components such as amplifiers, phase shifters, low pass filters, attenuators, and splitters add noise to the system. Through testing, criteria for the optimal performance of a system can be deduced.

\section{Materials and Methods}

This experiment consists of three main setups; the first provides information about the phase difference to voltage output sensitivity of each mixer, the second tests different combinations of components and determines the noise levels associated with each, and the third determines the phase drift associated with temperature changes for each component. The first experiment serves the purpose of gathering sensitivity data necessary to evaluate the noise and drift effects determined in the second and third experiments. This type of data are not specified by the vendors, as these effects only become appreciable when femtosecond time precision is desired; precision at this scale was unattainable before the LCLS was turned on.

\subsection{Sensitivity in Mixers}

As double-balanced mixers are currently being used to make phase measurements in the LCLS, these are the responsible for determining the sensitivity of phase timing. When two input signals are received by a mixer, through the Local Oscillator (LO) Port and the Radio Frequency (RF) Port, they are processed through a ring diode setup and an output signal is seen from the Intermed iate Frequency (IF) Port. A typical Schottky diode can be described by the equation:

$$
I=a_{1} V+a_{2} V^{2}+a_{3} V^{3}+a_{4} V^{4}+\cdots
$$

When this diode is excited by two sinusoidal waves, $\operatorname{Cos}\left(\omega_{1} t\right)$ and $\operatorname{Cos}\left(\omega_{2} t\right)$, as in the mixer, the equation thus becomes:

$$
I=a_{1}\left(\operatorname{Cos}\left(\omega_{1} \mathrm{t}\right)+\operatorname{Cos}\left(\omega_{2} \mathrm{t}\right)\right)+a_{2}\left(\operatorname{Cos}\left(\omega_{1} \mathrm{t}\right)+\operatorname{Cos}\left(\omega_{2} \mathrm{t}\right)\right)^{2}+\cdots
$$


When this equation is expanded, it contains the term " $2 a_{2} \operatorname{Cos}\left(\omega_{1} t\right) \operatorname{Cos}\left(\omega_{2} t\right)$ " which has the trigonometric relationship below:

$$
2 a_{2} \operatorname{Cos}\left(\omega_{1} t\right) \operatorname{Cos}\left(\omega_{2} t\right)=\operatorname{Cos}\left(\left(\omega_{1}-\omega_{2}\right) t\right)+\operatorname{Cos}\left(\left(\omega_{1}+\omega_{2}\right) t\right)
$$

This is defined as the output of mixing. ${ }^{[2]}$ The two sinusoidal waves describe the LO and RF inputs. The first and second terms on the right side of Equation 3 describe the difference in frequency of the two input signals, and the sum of their frequencies, respectively. Since we are only interested in the difference frequency, the higher frequency term is removed by a low pass filter, as seen in Figure 1.

Three mixers were tested to determine the picoseconds per volt phase difference, which is a measure of the mixers' sensitivities. The Mini-Circuits mixers used were models ZX05-HWS+, ZFM-4H-S+, and ZFM-150. Two signal generators, Hewlett Packard E4432B ESG-D Series and Hewlett Packard 8656B, were used to generate LO and RF inputs respectively. The RF was set at $476 \mathrm{MHz}$ and the $\mathrm{LO}$ at $476.01 \mathrm{MHz}$ to generate at $10 \mathrm{kHz}$ IF output. Both signals passed through a Mini-Circuits VLFX-470 Low Pass Filter with a passband from DC to $470 \mathrm{MHz}$. While this low pass filter slightly attenuated the primary signal, it served to eliminate higher level harmonics from creating unwanted signals and noise in the mixer. By removing these harmonics, unwanted IF products were also eliminated and the observed noise floor examined in the second part of the experiment was lowered.

Sensitivity readings are taken with LO powers of $5,10,15,17$, and $20 \mathrm{dBm}$ for the first two listed mixers along with an additional $0 \mathrm{dBm}$ reading taken for the third due to its lower power level specifications for LO inputs. At each of these levels, RF inputs of $0,5,10$, and 15 $\mathrm{dBm}$ were used. As cables have some power loss, a Mini-Circuits PWR-SEN-6GHS+ Power Sensor was used before every measurement right before the input ports to assure the correct power level were entering the mixer. This array of data was taken as such to allow for interpolation of sensitivities for the next parts of the experiment. 
The IF output of the mixer is sent through a Mini-Circuits SLP-100+ Low Pass Filter with a passband from DC to $98 \mathrm{MHz}$ to eliminate the summation frequency and only allow the difference frequency to be analyzed in the Tektronix TDS3032 Oscilloscope. With the oscilloscope, the $10 \mathrm{kHz}$ wave for each combination of power inputs is measured for its voltage maximum and minimums and more importantly its sensitivity. To determine phase with a mixer, it is preferable to use the portion of the IF that crosses directly at the zero voltage mark. When the signals are in phase or 180 degrees out of phase, the IF signals are at a maximum or minimum. Around these points, when the phases shift slightly, the corresponding voltage change is small and often undetectable; only changes in power input can be seen as a change in amplitude. When the signals are 90 degrees out of phase, the sinusoidal waves slope is the greatest. Here, the slightest phase shift results in a large voltage change and power input changes will theoretically not affect the sensitivity. By zooming into the zero-intercept on the oscilloscope and measuring the voltage change per time slope, one measures the voltage change produced by a specified phase shift between the two signals. A visual representation of what this actual looks like on the oscilloscopes can be seen in Figure 2. As we know the two signals shift one full cycle in phase at the IF frequency, the observed data can be converted to volts per cycle for the IF, shown in Equation 4 below. Due to the IF frequency's dependence upon the RF frequency, we can finally then convert this to a picoseconds per volt phase shift for the mixer and initial RF, a measure of sensitivity, shown in Equation 5.

$$
\begin{aligned}
& \text { Volts/Cycle }[\mathrm{V}]=\text { Slope }[\mathrm{V} / \mathrm{s}] *\left(1 / f_{\mathrm{IF}}\right)[\mathrm{s}] \\
& \text { Sensitivity }[\mathrm{V} / \mathrm{ps}]=\text { Volts } / \text { Cycle }[\mathrm{V}] * \boldsymbol{f}_{\mathrm{RF}}\left[\mathrm{s}^{-1}\right]
\end{aligned}
$$




\subsection{Noise Measurements}

When trying to optimize a phase detection system, mixer sensitivity alone cannot be used; a ratio of sensitivity to noise must be obtained and analyzed. To measure sensitivity a single RF source is used, as seen in Figure 3. The Hewlett Packard E4432B ESG-D Series Signal Generator is set to $476 \mathrm{MHz}$, the frequency of the master oscillator for the LCLS. Due to the maximum power of the signal generator being 15dBm, a Mini-Circuits ZHL-2010+ Low Noise Amplifier was placed between the RF source and the low pass filter to obtain the power levels needed to examine a wider range of LO and RF inputs. The low pass filter served to remove any harmonics created by the amplifier. Next, the signal was split by either a Mini-Circuits ZFSC-21W+ power splitter or an Anaren 40263 strip-line power splitter. Both were tested to determine differences between magnetic core splitters and strip line splitters. For noise experiments a splitter is used in order to allow both the RF and LO inputs to have exactly the same frequency signal, therefore producing a DC voltage IF. The RF signal is passed through an Aeroflex Weinschel Model 980 Mechanical Phase Shifter in order to adjust the phase difference created by the difference in path lengths leading to the RF and LO ports.

Before noise measurements are taken, the IF output is led into a Fluke 289 True RMS Multimeter. The phase shifter then adjusts the DC output on the voltmeter to read 0 Volts, thereby setting the two inputs 90 degrees out of phase. This sets the mixer at the position of highest sensitivity, which, in practice, will be where phase detection is done. Next, the signal is fed into an Agilent 89441 A Vector Signal Analyzer for noise level readings. The power levels these measurements are taken at are LO matches to the sensitivity reading levels while the RF readings were taken as close as possible by use of attenuators before the RF port. Any differences were accounted for by interpolation between sensitivity data. For each power level, the noise floor was determined at four different bandwidths of $100 \mathrm{~Hz}, 1 \mathrm{kHz}, 10 \mathrm{kHz}$, and 100 $\mathrm{kHz}$. The noise floor voltage divided by the square root of the bandwidth gives a measure of 
volts per square root hertz. The noise per root bandwidth over the sensitivity will finally give a noise per sensitivity ratio, which can be used to determine optimization information for the mixers and splitters.

\subsection{Temperature Drift}

Another factor to consider when choosing components is the DC output voltage drift per temperature change. To determine this temperature drift, the same setup as seen in Figure 3 was used, except the component being tested was placed on a temperature controlled plate. The plate containing the rest of the components was monitored for temperature changes as well. The temperature range for each component varied but was usually between $14^{\circ} \mathrm{C}$ and $30^{\circ} \mathrm{C}$. As done previously in the noise measurements, the signal was fed into the Fluke 289 True RMS Multimeter and set to zero volts output by the phase shifter. However, this time it was done specifically at the top of the temperature range. After being zeroed, the tempera ture controlled plate and the component being tested were lowered to the bottom of the range, using a Merlin M33 Series chiller. The phase drift, seen as a DC output voltage, was recorded at each integer interval over the temperature range. Without resetting the phase difference, the temperature was increased back to its initial state as readings were taken again.

Two observations about the temperature drift are demonstrated by these measurements.

First, if the DC output voltage did not return back to zero, some change in the system most likely occurred and the test loses some validity. Secondly, the average DC voltage change per degree Celsius corresponds directly to the picoseconds phase drift between the two signals. This phase drift per degree Celsius, along with the noise measurements, create two important parameters for choosing optimal components in a high-precision phase-detection system. 


\section{Results}

The sensitivity readings, represented as voltage output per picosecond phase difference can be seen in Figures 4, 5, and 6. Graphing RF input powers against LO input powers give a better visual representation of the most sensitive power inputs and moreover, regions where the sensitivity does not change significantly over different input ranges. The three mixers, MiniCircuits Models ZX05-HW-S+, ZFM-4H-S+, and ZFM-150, all had the highest sensitivities at the tested inputs of $20 \mathrm{dBm} \mathrm{LO}$ and $15 \mathrm{dBm} R F$ with values of $0.05620 \mathrm{~V} / \mathrm{ps}, 0.04430 \mathrm{~V} / \mathrm{ps}$, and $0.00897 \mathrm{~V} / \mathrm{ps}$ respectively. A plateau was also seen above each mixer's recommended LO input, 17dBm for models ZX05-HW-S+ and ZFM-4H-S+, 10dBm for model ZFM-150. Model ZX05HW-S+'s plateau was above its recommended LO value, where the previous sensitivity trend disappeared. Over the range from $17-20 \mathrm{dBm}$, this mixer saw on average, a change of less than $0.000592 \mathrm{~V} / \mathrm{ps}$. The model ZFM-4H-S+ did not have as distinct a plateau over the recommended LO input; however, the sensitivity trend's slope did decrease considerably. Over the range from 17-20 dBm, on average, sensitivity changes were less than $0.00119 \mathrm{~V} / \mathrm{ps}$. The model ZFM-150's plateau was much broader as the recommended LO input was $10 \mathrm{dBm}$ so more readings were taken above its saturated value than for the other mixers. The third mixers plateau spans from 10 to $15 \mathrm{dBm}$ LO input and changes on average less than $0.00016 \mathrm{~V} / \mathrm{ps}$.

Each specific combination of components for the second experiment was measured for a volts per root hertz noise floor at four bandwidths. These were then divided by the sensitivities calculated earlier for each mixer to determine a no ise to sensitivity ratio, NSR, with unit's picoseconds per root hertz. Table 1 contains the average NSR over the four bandwidths for different power inputs for all setups, as well as the minimum NSR value for each. Table 2 compares the input power levels for the optimal NSR and sensitivity.

When individual components of the second experimental setup were placed on a temperature controlled plate for the third experiment, smooth linear changes in the DC offset 
were observed. The DC offset change between each degree Celsius change was taken and averaged to arrive at the temperature drift readings listed in Table 3. The ten foot Coaxial Cable reacted most with a $81.58 \mathrm{fs} /{ }^{0} \mathrm{C}$ phase drift. While not the most sensitive, or the least noisy, the second mixer showed the least temperature drift with a value of $1.6949 \mathrm{fs} /{ }^{0} \mathrm{C}$.

\subsection{Discussion-Sensitivity}

From the sensitivity data, the most useful feature is not the maximum sensitivity reading, but the plateau that exists above particular LO inputs, as well as a constant rise in sensitivity for RF inputs. If one's goal is to increase the sensitivity of a mixer to its maximum, applying the largest RF input power the mixer can withstand would be the way to achieve this. However, applying a LO input power above the recommended LO input does not change the sensitivity appreciably. This effect is due to saturation of the diodes in the mixer. Above this power level, the mixer acts as a limiter and increases to the LO power input do not affect the IF output. Operation in this saturated range is optimal for users wishing to keep a consistent sensitivity value when their system contains sources of small amplitude fluctuations.

\subsection{Component Noise}

While the sensitivity is a useful measurement when choosing a mixer for normal operation, at the LCLS, precision measurements are often limited by the noise associated with each setup. The two types of noise investigated in this project were white and pink noise. White noise is consistent over the entire frequency spectrum and created by phonons, or thermal vibrations, and impurities in the conductors. White noise can only be eliminated so much by temperature control until very expensive and exotic means are necessary to transfer the signal through the temperature change without signal distortion. Pink noise exhibits a $1 / \mathrm{f}$ spectrum (equal power per decade of frequency). ${ }^{[3]}$ The noise measurements were taken at a minimum bandwid th of $100 \mathrm{~Hz}$ with a range of $0-10 \mathrm{kHz}$. What is seen as pink noise at this bandwidth and higher band widths can actually be resolved by using a $10 \mathrm{~Hz}$ bandwidth. When a $10 \mathrm{~Hz}$ 
bandwidth was used, the $0-1 \mathrm{kHz}$ range was full of external power source signals at $60 \mathrm{~Hz}$ and appreciable harmonics of such as high as $600 \mathrm{~Hz}$. From this finding, it can be seen that these systems have no significant pink noise. Due to this, all noise measurements are based strictly on the white noise floor.

Beginning with the comparison of the two types of splitters, one made of magnetic circuitry and ferrite cores, the other which is a strip-line splitter etched into a silicon base plate, while both produced similar NSR values, the minimum value for each mixer existed when the magnetic circuitry mixer was used. This trend also exists across the input power spectrum where the NSR is not at its minimum. Differences in noise levels could be explained by construct, such as impurities and stability of internal components.

As for the mixers, while each had similar minimum noise values between 5.70 and 5.85 $\mathrm{nV} / \sqrt{\mathrm{Hz}}$, the sensitivities made the clear distinction between their NSR's. The minimum NSR's for the mixers were valued from $1.69^{*} 10^{-7}$ to $9.16^{*} 10^{-7} \mathrm{ps} / \sqrt{\mathrm{Hz}}$, showing the importance of sensitivity when choosing a mixer. With measurement differences of almost half an order of magnitude among the different mixers, it is apparent that, while the effect is not tremendous, when dealing with mixers in a precision detection system, such as the LCLS, factors such as noise must be tested and taken into account.

For completion, a $20 \mathrm{~dB}$ amplifier followed by a $20 \mathrm{~dB}$ attenuator was placed between the phase shifter and RF input of the mixer. Noise levels were taken with and without this combination to see how much noise was being added to the system by the amplifier. As it turned out, the amplifiers used in this experiment added no noticeable noise. 


\subsection{Temperature Drift}

Components such as the mechanical phase shifter and coaxial cable created the largest temperature drift because they contained the longest metal path length, making them most susceptible to expansion with temperature. Not surprisingly, the strip-line splitter's temperature drift was more than twice the magnetic splitter's drift. For unknown reasons, certain mixers were more than an order of magnitude less susceptible to temperature drift. It is presumed answers may lie within the details of the components construction, details withheld by the vendors.

\subsection{Conclusion}

When designing a highly precise system for making femtosecond phase measurements, it is impractical to build small components such as mixers for such a large project as the LCLS. As these components are not created for the specifications needed, one must judge how important parameters, such as noise levels and temperature stability, are to the project. With the optimal components and parameters, taking a measurement at $200 \mathrm{kHz}$, typical of LCLS, a timing noise of $0.10420 \mathrm{fs}$ is observed. Combined with the $0.16948 \mathrm{fs}$ timing drift, calculated with the assumption of one-tenth degree Celsius temperature stability, the best combined timing noise for the tested systems is $0.27368 \mathrm{fs}$. This is an order of magnitude lower than the noise currently seen at the LCLS, implying that with attention given to component testing and analysis, precision of the LCLS phase detection systems can be improved in the future.

\section{Acknowledgements}

This research was carried out at the SLAC National Accelerator Laboratory between June 21 and August 14, 2010 under the direction of Josef Frisch. I would like to thank the United States Department of Energy, SLAC National Laboratory, and the directors and administrators of the SULI program for making this research possible. I would like to thank my mentor Josef Frisch for his enthusiasm and guidance. I would also like to thank George Burgueno for his help 
with procuring the tools necessary to carrying out this research. I would like to thank Cara Perkins for working side by side with me, allowing for all of this research to have been completed.

\section{References}

[1] A. Brachmann, et a1,. Femtosecond Operation of the LCLS for User Experiments: TUPE066 International Particle Accelerator Conference, May 23-28, 2010, Kyoto, Japan.

[2] Devlin Liam “Mixers," Plextek Communications Technology Consultants, July 17, 2003. [Online]. Availible: http://www.plextek.co.uk/papers/mixers2.pdf. [Accessed: July 20, 2010]. [3] P. Horowitz and W. Hill, The Art of Electronics, $2^{\text {nd }}$ ed. Cambridge: Cambridge University Press, 1989, pp. 430-432. 


\section{Figures and Tables}

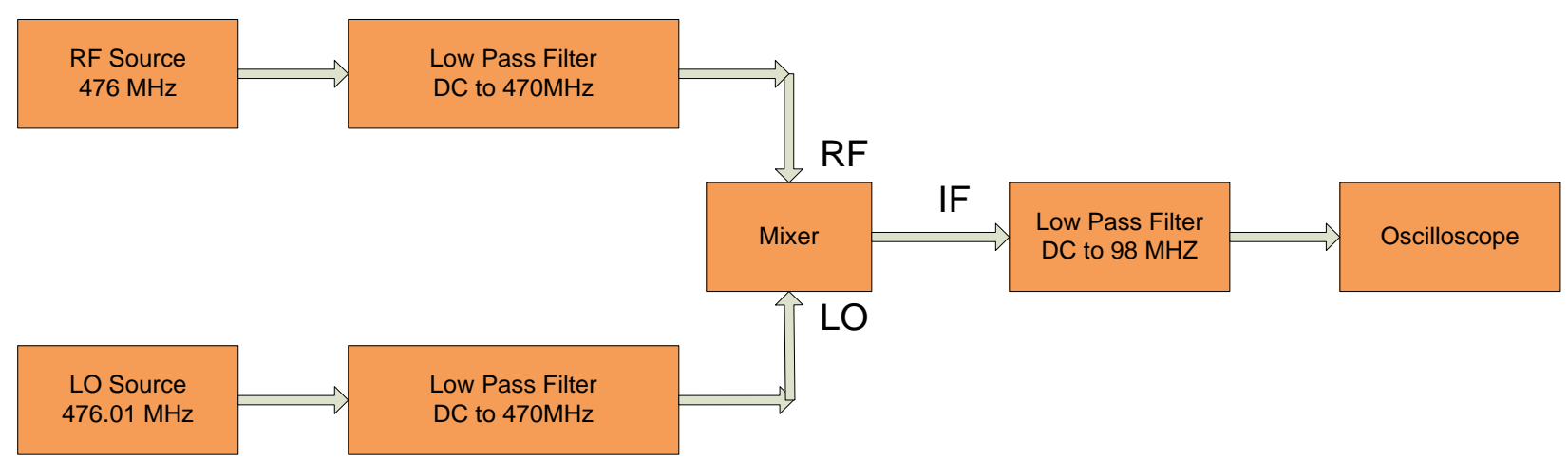

Figure 1: Sensitivity Measurement Setup

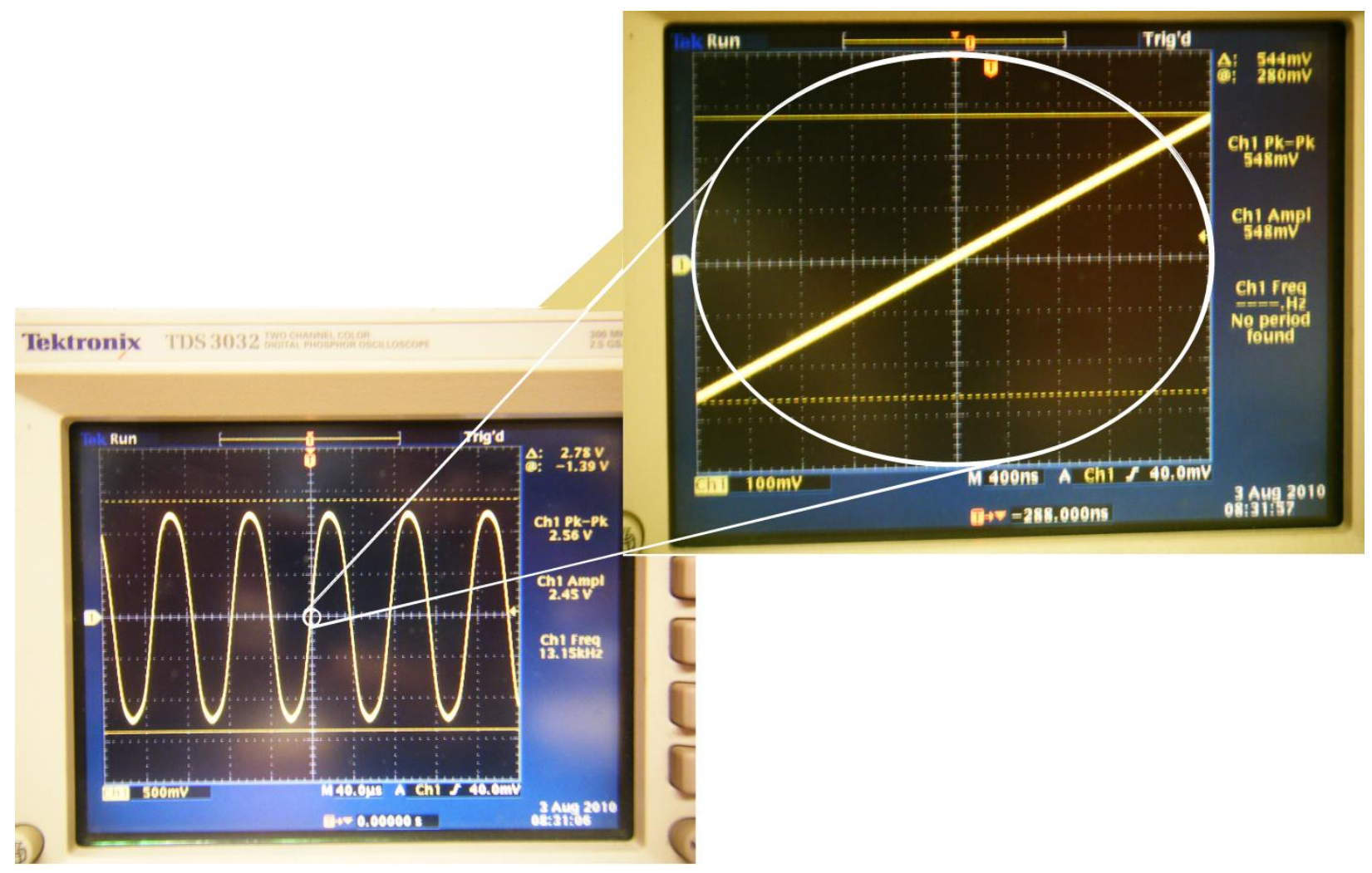

Figure 2: Obtaining data at zero-intercept for maximum sensitivity 


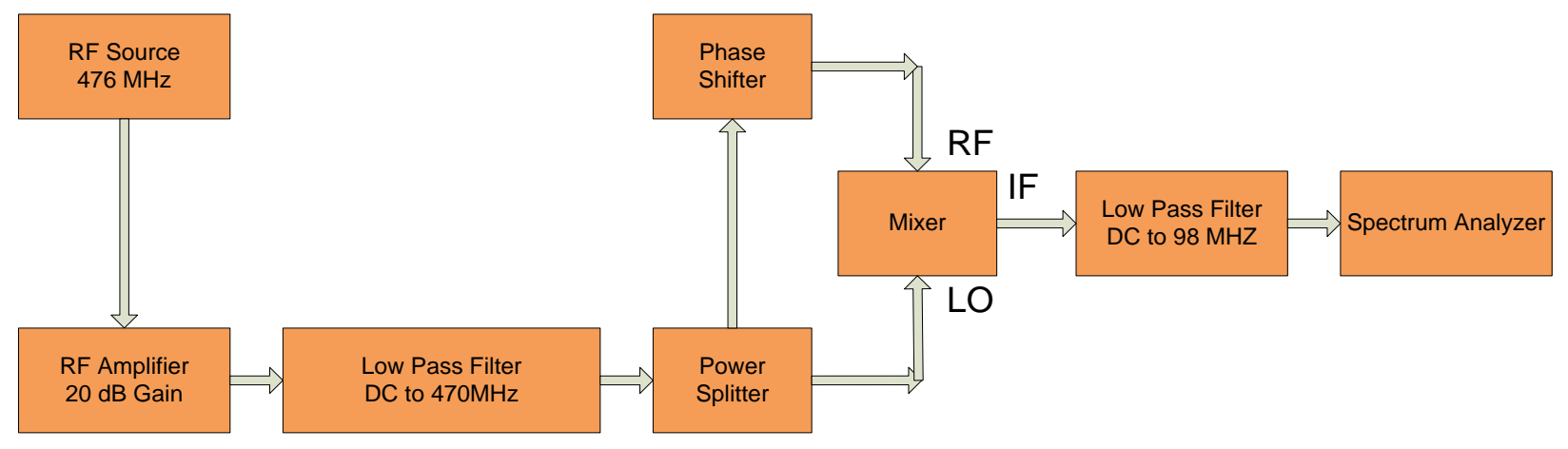

Figure 3: Noise and Drift Measurement Setup

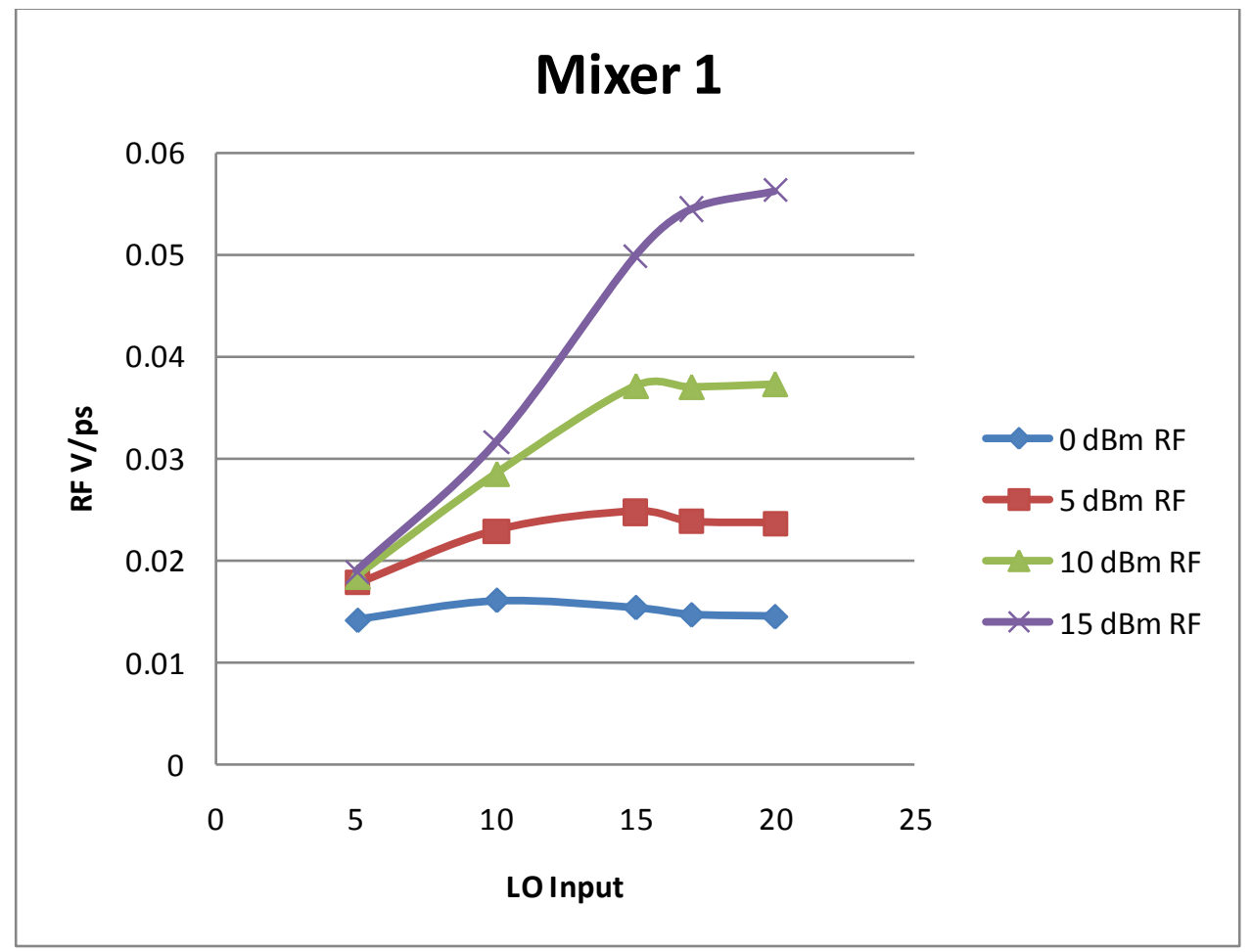

Figure 4: Model ZX05-HW-S+ mixer sensitivity graph 


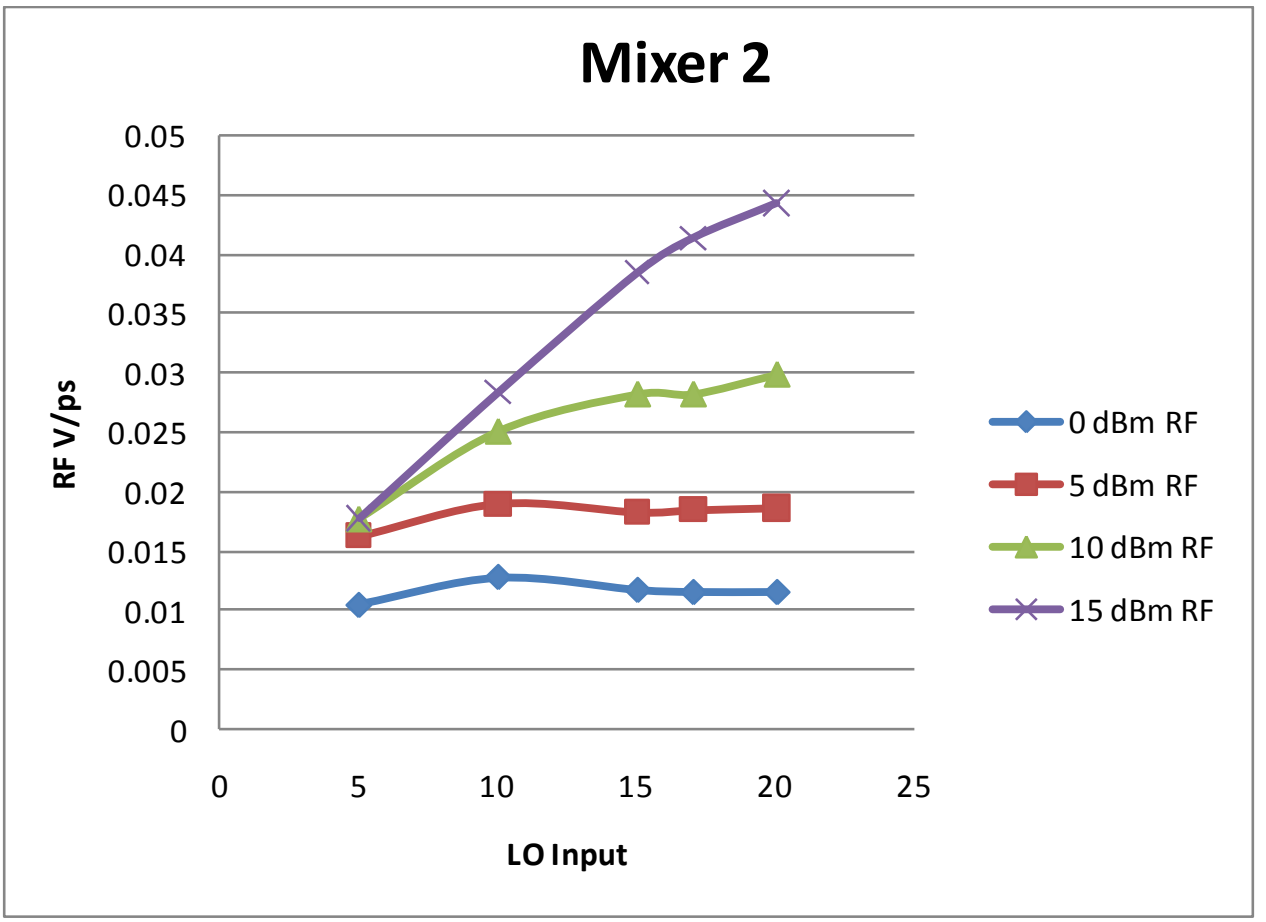

Figure 5: Model ZFM-4H-S+ mixer sensitivity graph

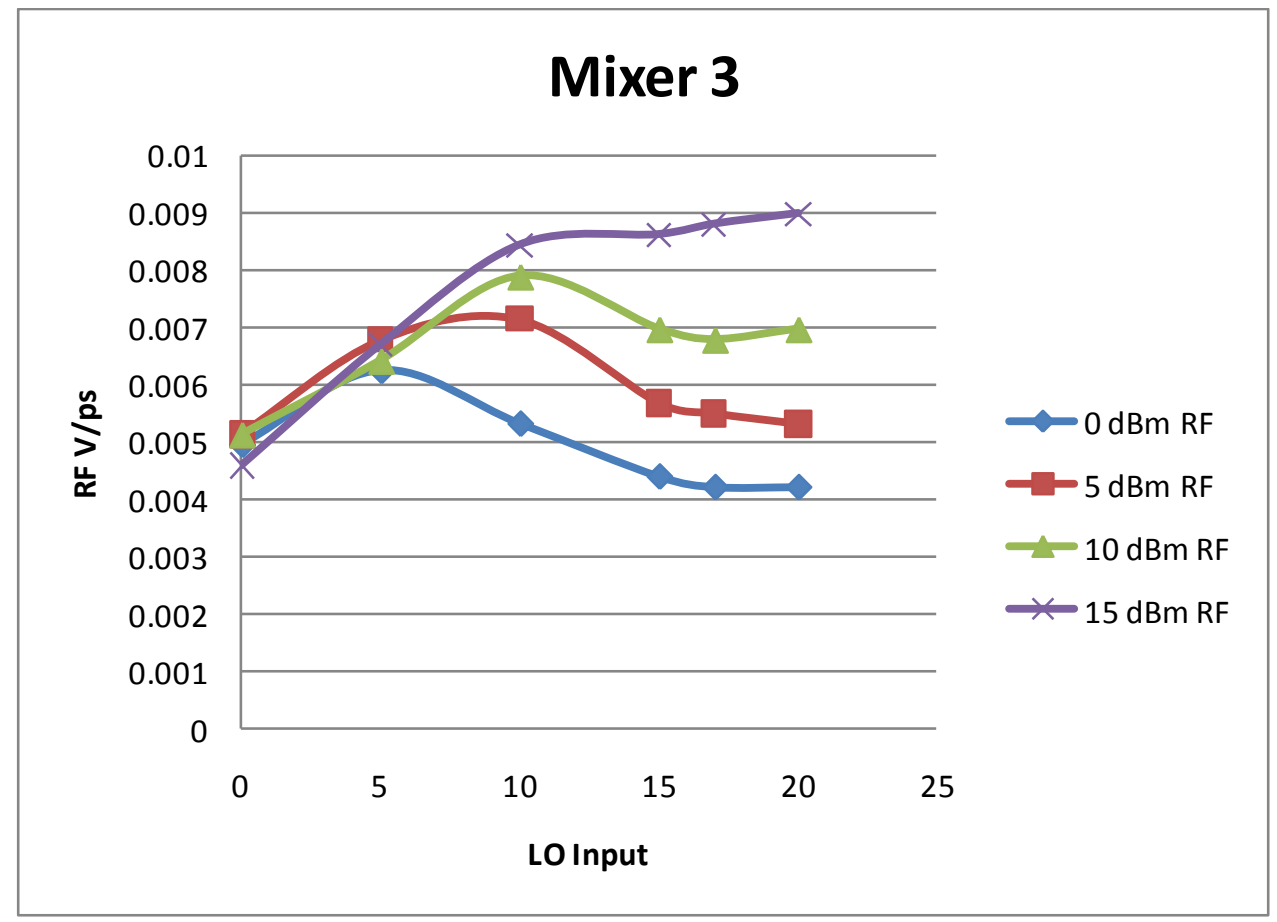

Figure 6: Model ZFM-150 mixer sensitivity graph 


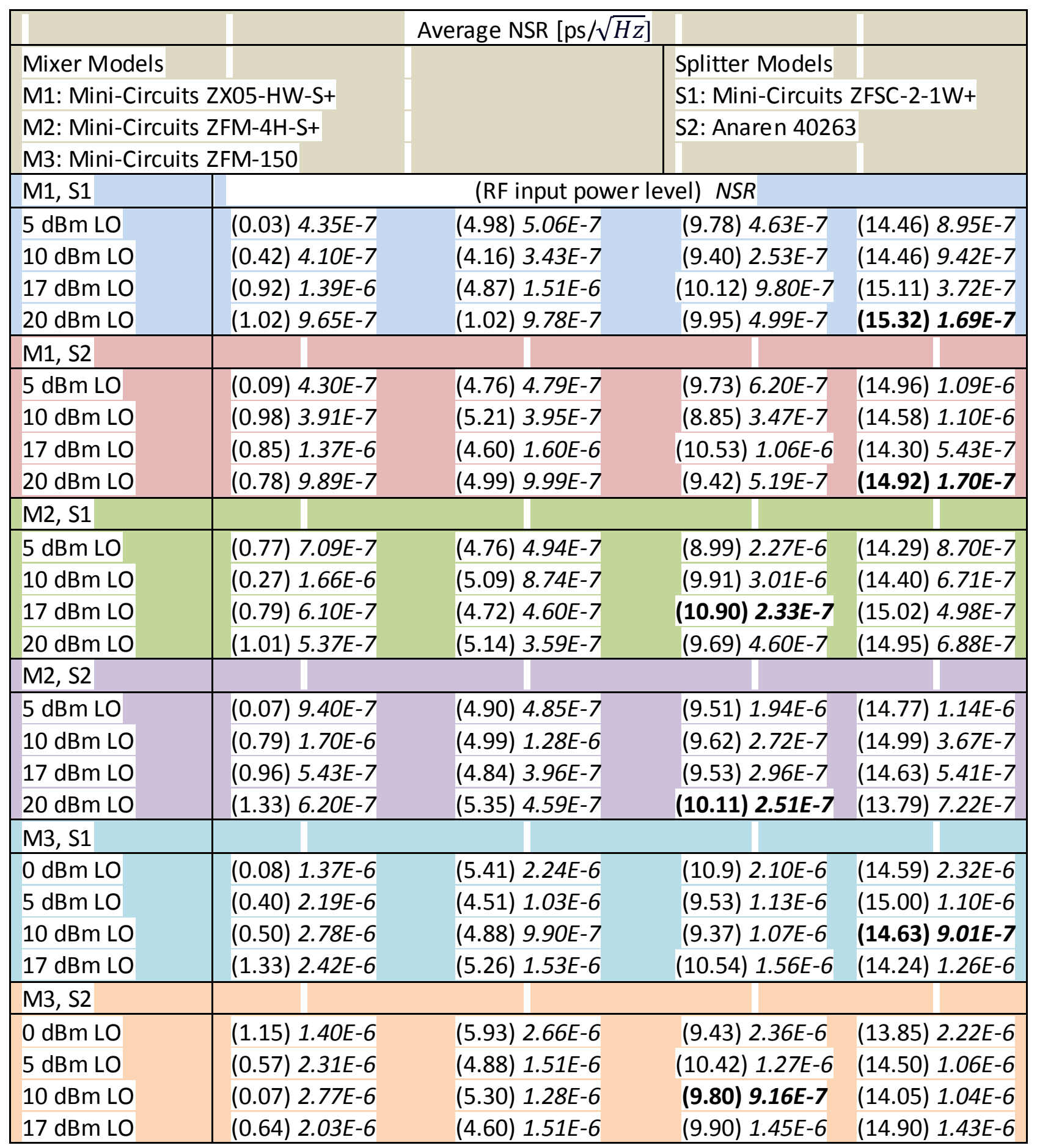

Table 1: Average NSR for all mixer, splitter, LO and RF power level combinations (Minimum value for each component combination highlighted in bold.) 


\begin{tabular}{|c|c|c|}
\hline \multicolumn{2}{|c|}{$\begin{array}{l}\text { Mixer Models } \\
\text { M1: Mini-Circuits ZX05-HW-S+ } \\
\text { M2: Mini-Circuits ZFM-4H-S+ } \\
\text { M3: Mini-Circuits ZFM-150 }\end{array}$} & $\begin{array}{l}\text { Splitter Models } \\
\text { S1: Mini-Circuits ZFSC-2- } \\
\text { 1W+ } \\
\text { S2: Anaren } 40263\end{array}$ \\
\hline & $\begin{array}{l}\text { Minimum NSR (LO, } \\
\text { RF) }\end{array}$ & $\begin{array}{l}\text { Maximum Sensitivity (LO, } \\
\text { RF) }\end{array}$ \\
\hline $\mathrm{M} 1, \mathrm{~S} 1$ & $(20 \mathrm{dBm}, 15.32 \mathrm{dBm})$ & $(20 \mathrm{dBm}, 15 \mathrm{dBm})$ \\
\hline $\mathrm{M} 1, \mathrm{~S} 2$ & (20 dBm, $14.92 \mathrm{dBm})$ & (20 dBm, $15 \mathrm{dBm})$ \\
\hline $\mathrm{M} 2, \mathrm{~S} 1$ & $(17 \mathrm{dBm}, 10.90 \mathrm{dBm})$ & (20 dBm, $15 \mathrm{dBm})$ \\
\hline $\mathrm{M} 2, \mathrm{~S} 2$ & $(20 \mathrm{dBm}, 10.11 \mathrm{dBm})$ & (20 dBm, $15 \mathrm{dBm})$ \\
\hline M3, S1 & $(10 \mathrm{dBm}, 14.63 \mathrm{dBm})$ & (20 dBm, $15 \mathrm{dBm})$ \\
\hline M3, S2 & $(10 \mathrm{dBm}, 9.80 \mathrm{dBm})$ & (20 dBm, $15 \mathrm{dBm})$ \\
\hline
\end{tabular}

Table 2: Comparison of minimum NSR and maximum Sensitivity power level inputs

\begin{tabular}{|l|r|}
\hline Component & \multicolumn{2}{|c|}{$\begin{array}{l}\text { Temperature Drift } \\
\left(\mathrm{fs} /{ }^{\circ} \mathrm{C}\right)\end{array}$} \\
\hline Mini-Circuits Splitter Model ZFSC-2-1W+ & 3.780594406 \\
\hline Anaren Splitter Model 40263 & 8.503419973 \\
Mini-Circuits Mixer Model ZX05-HW-S+ & 26.79407713 \\
Mini-Circuits Mixer Model ZFM-4H-S+ & 1.694897959 \\
Mini-Circuits Mixer Model ZFM-150 & 6.952019732 \\
Aeroflex Phase Shifter Model 980 & 24.56293706 \\
\hline $10^{\prime}$ Belden Coaxial 50 Ohm Microwave Cable Model & 81.58277672 \\
$1673 \mathrm{~A}$ & \\
\hline
\end{tabular}

Table 3: Temperature Drift 